\title{
Social-Group-Optimization based Tumor Evaluation Tool for Clinical Brain MRI of Flair/ Diffusion-Weighted Modality
}

Nilanjan Dey¹, V. Rajinikanth², Fuqian Shi ${ }^{3}$, João Manuel R. S. Tavares ${ }^{4}$, Luminita Moraru ${ }^{5}$, $\quad$ K. Arvind Karthik ${ }^{6}$, Hong Lin ${ }^{7}$, K. Kamalanand ${ }^{8}$, C. Emmanuel ${ }^{9}$

${ }^{1}$ Department of Information Technology, Techno India College of Technology, Kolkata-700156, West Bengal, India; neelanjan.dey@gmail.com

${ }^{2}$ Department of Electronics and Instrumentation Engineering, St. Joseph's College of Engineering, Chennai-600 119, Tamilnadu, India; rajinikanthv@st.josephs.ac.in

${ }^{3}$ College of Information and Engineering, Wenzhou Medical University, Wenzhou 325035, China, sfq@wmu.edu.cn

${ }^{4}$ Instituto de Ciênciae Inovaçãoem Engenharia Mecânicae Engenharia Industrial, Departamento de Engenharia Mecânica, Faculdade de Engenharia, Universidade do Porto, Porto, Rua Dr. Roberto Frias, s/n, 4200-465 Porto, Portugal; tavares@fe.up.pt

${ }^{5}$ Dunărea de Jos, University of Galati, Faculty of Sciences and Environment, Department of Chemistry, Physics and Environment, 47 Domnească Street, RO-800008, Galati, Romania; luminita.moraru@ugal.ro

${ }^{6}$ Managing Director and Radiologist, Proscan Diagnostics Private Limited, Chennai-600 049, Tamilnadu, India; a_karthik15@hotmail.com

${ }^{7}$ Department of Computer Science and Engineering Technology, University of Houston-Downtown, 1 Main Street, Houston, Texas 77002-1001, USA; 1inh@uhd.edu

${ }^{8}$ Department of Instrumentation Engineering, MIT Campus, Anna University, Chennai - 600044, Tamilnadu, India; kamalanand@mitindia.edu

${ }^{9}$ Gleneagles Global Health City, Perumbakkam, Chennai - 600100, Tamilnadu, India; cyem69@yahoo.co.in

\begin{abstract}
Brain tumor is one of the harsh diseases among human community and is usually diagnosed with medical imaging procedures. Computed-Tomography (CT) and Magnetic-ResonanceImage (MRI) are the regularly used non-invasive methods to acquire brain abnormalities for medical study. Due to its importance, a significant quantity of image assessment and decision-making procedures exist in literature. This article proposes a two-stage image assessment tool to examine brain MR images acquired using the Flair and DW modalities. The combination of the Social-GroupOptimization (SGO) and Shannon's-Entropy (SE) supported multi-thresholding is implemented to pre-processing the input images. The image post-processing includes several procedures, such as Active Contour (AC), Watershed and region-growing segmentation, to extract the tumor section. Finally, a classifier system is implemented using ANFIS to categorize the tumor under analysis into benign and malignant. Experimental investigation was executed using benchmark datasets, like ISLES and BRATS, and also clinical MR images obtained with Flair/DW modality. The outcome of this study confirms that AC offers enhanced results compared with other segmentation procedures considered in this article. The ANFIS classifier obtained an accuracy of $94.51 \%$ on the used ISLES and real clinical images.
\end{abstract}


Keywords: Brain MRI, Tumor, Social group optimization, Shannon's entropy, ANFIS classifier, Clinical MRI dataset. 


\title{
Social-Group-Optimization based Tumor Evaluation Tool for Clinical Brain MRI of Flair/DW Modality
}

\begin{abstract}
Brain tumor is one of the harsh diseases among human community and is usually diagnosed with medical imaging procedures. Computed-Tomography (CT) and Magnetic-ResonanceImage (MRI) are the regularly used non-invasive methods to acquire brain abnormalities for medical study. Due to its importance, a significant quantity of image assessment and decision-making procedures exist in literature. This article proposes a two-stage image assessment tool to examine brain MR images acquired using the Flair and DW modalities. The combination of the Social-GroupOptimization (SGO) and Shannon's-Entropy (SE) supported multi-thresholding is implemented to pre-processing the input images. The image post-processing includes several procedures, such as Active Contour (AC), Watershed and region-growing segmentation, to extract the tumor section. Finally, a classifier system is implemented using ANFIS to categorize the tumor under analysis into benign and malignant. Experimental investigation was executed using benchmark datasets, like ISLES and BRATS, and also clinical MR images obtained with Flair/DW modality. The outcome of this study confirms that AC offers enhanced results compared with other segmentation procedures considered in this article. The ANFIS classifier obtained an accuracy of $94.51 \%$ on the used ISLES and real clinical images.
\end{abstract}

Keywords: Brain MRI, Tumor, Social group optimization, Shannon's entropy, ANFIS classifier, Clinical MRI dataset.

\section{Introduction}

Brain is the most significant internal organ, responsible to supervise the complete operation in human body by interpreting the information gathered from other body regions. The foremost infirmity in brain, such as stroke and tumor, may cause moderate to harsh impact in patient's activity. A well planned medical examination and a competent diagnosis are essential to identify the location and severity of brain abnormality to plan for an appropriate treatment procedure. Common healing approaches, such as chemotherapy and surgery, are widely adopted to treat the most common brain illness called tumor. Benign tumors are less hazardous and absolutely curable with precise surgery and chemotherapy. Malignant tumors are dangerous and require extraordinary consideration by the surgeon during the examination as well as treatment planning.

Brain abnormality evaluation involves in the implementation of dedicated hardware systems to acquire essential brain information based on brain-signals and brain-images in a controlled environment. Earlier research confirms that imaging based activities offer more apparent information on brain irregularity compared to the signal supported assessment [1-3]. Mapping of brain signal along with the brain image is also a flourishing research field [4]. To have superior perceptive on brain irregularity, it is essential to consider imaging procedures, such as Magnetic Resonance Imaging (MRI), Computed Tomography (CT) and Positron Emission Tomography (PET), which are widely adopted in clinics [5-7]. PET is a nuclear imaging procedure normally used along with CT. MRI is one of the widely used in medical domain to acquire images of internal body parts. It supports a varied group of reconstructed three-dimensional (3D) images of Flair, T1, T1C, T2 and DiffusionWeight (DW) [8]. Later, based on the necessity and availability of the diagnosing procedures, these images can be examined in the form of $3 \mathrm{D}$ or $2 \mathrm{D}$ images. The examination of $3 \mathrm{D}$ is quite complex and require more computation time. Hence, in recent years, 2D MRI examination is widely accepted 
by the researchers to inspect MRI exams obtained according to universal configurations, such as axial, sagittal and coronal $[9,10]$.

The existing works on brain MRI confirms the availability of traditional machine learning and deep learning based approaches implemented and evaluated on user defined and benchmark datasets $[11,12]$. The aim of this research work was to develop a Computerized Disease inspection Tool (CDT) using a Hybrid Image Processing (HIP) procedure recently discussed in the literature [1-3]. HIP was developed by integrating a pre-processing practice based on the Social-Group-Optimization (SGO) assisted Shannon's thresholding and a post-processing based on Active-Contour, Marker-ControlledWatershed and Seed-Region-Growing procedures to segment the suspicious region. The proposed CDT was initially tested on well-known benchmark MRI datasets, such as ISLES2015 [13-15] and BRATS $[16,17]$, and then evaluated with real clinical brain MRI scans acquired with Flair and DW modalities.

The main inspiration behind the proposed HIP was to build a soft-computing assisted image processing tool to examine MR images with better accuracy. For real time applications, it is compulsory to include a unique MRI diagnosing tool that can assist doctors to examine the brain images acquired with various modalities with high efficiency. This article also addresses common MRI issues, such as skull stripping, image re-scaling, choice of the segmentation method, and difficulties in classification. The proposed solution was implemented in Matlab software (Version 7, Release 14, License number 285705 with perpetual term, St. Joseph's College of Engineering, Chennai, India), and the experimental findings confirm that it offers promising result on the used benchmark datasets as well as real on clinical brain images.

\section{Related Earlier Works}

MRI is extensively considered in medical field to assess activities and abnormalities of internal organs. The advancements in the MRI scanners support furthermore the rebuilding and assessment of 3D digital images. Existing assessment schemes for these images are more complex and need high speed computing devices because of its large data volume. Usually, to minimize the complexity during the MRI examination, restructured 3D images are then converted into a considerable number of $2 \mathrm{D}$ images, i.e. slices, in order to employ an appropriate image examination scheme. The earlier investigation on brain MRI considers both the 3D and 2D images for abnormality estimation. However, 3D brain MRI evaluation procedures are still very limited due to its computational difficulty. Jiang et al. proposed learning population based 3D brain tumor segmentation procedure on real time MR images [18]. Song et al. developed a comparison study on clinical brain segmentation with 3D MRI [19]. Maria et al. discussed about the registration of 3D fetal MRI [20].

CDT supported 2D brain slice assessment is extensively discussed by the researchers because of its simplicity, less run time, and enhanced accuracy. Recently, automated and semi-automated MR image examination procedures are employed to find brain abnormalities, like tumor and stroke sections, from various MRI modalities. Palani et al. discussed a two-step procedure based on the Otsu's thresholding and Markov-Random-Field (MRF) segmentation procedure to examine brain images with and without skull section [1]. Similar approach with MRF is extended using the Firefly Algorithm (FA) by Rajinikanth et al. [10] and implemented to extract the tumor section from Flair, T1, and T2 modalities of MRI. The research work by Rajinikanth et al. provides a detailed evaluation of various entropy based thresholding approaches on the BRATS dataset and confirmed that Shannon's entropy based procedure offers enhanced results for tumor enhancement [2]. Rajinikanth et al. proposed a Cuckoo Search (CS) approach to examine 2D MRI using the Tsallis entropy and DRLS segmentation [3]. Roopini et al. proposed a two stages scheme based on the Fuzzy entropy and DRLS approach to extract and examine the tumor section of CEREBRIX and BRAINIX databases [21]. The 
work of Rajinikanth and Satapathy [8] implements a hybrid approach based on the Social Group Optimization (SGO) and Fuzzy-Tsallis entropy to examine the stroke region from the MRI of ISLES dataset. This work also provides a detailed comparative assessment on various tumor segmentation procedures existing in the literature. Rajinikanth et al. proposed an image fusion approach to enhance the outcome during the MRI examination [22]. In their work, the BRATS dataset is considered for the examination and the fusion approach is considered to attain images like Flair+T1, Flair+T2, Flair+T1C and Flair+T2+T1C. This study confirms that the image fusion approach facilitates superior image performance measures compared with the MRI with single modality. Kanmani and Marikkannu implemented a tumor mining and classification approach which obtained better statistical measures [5]. Nilesh et al. proposed a detailed evaluation of brain tumor examination and classification procedure for brain MRI analysis [6]. Marzena and Khalid discussed a segmentation and localization procedure for brain tumor [7]. Bhattacharyya and Kim implemented an ANN scheme to detect tumor from MRI [9]. Rajinikanth et al. implemented the Tsallis entropy and the Active Contour (AC) segmentation approach to examine MR angiograms and MRI of BRATS [23]. This work also confirms that $\mathrm{AC}$ is a semi-automated segmentation procedure and offer better results for tumor extraction and evaluation. Isin et al. provided a brief review about MRI tumor extraction with deep learning techniques [11]. The research of Usman and Rajpoot [12] gives a detailed discussion on extracting tumor sections from multi-modality MRI. This work considered the BRATS 2013 dataset and achieved $88 \%$ for Dice coefficient. Further, the brain tumor examination procedures can also be found in [24-32].

The objective of this article is to present an inspection scheme for the MRI acquired with various modalities. Furthermore, this work addresses the need of skull stripping, thresholding, segmentation, tumor feature extraction and selection practices. It also discusses the complexity in employing the classifier scheme for a brain MRI dataset.

\section{Methodology}

This section provides an overview regarding the procedures considered in this work to develop a CDT for brain MRI analysis. Then, the database considered, the initial task in brain image assessment, pre-processing, post-processing, segmentation, tumor feature extraction and classification are discussed in detail. 


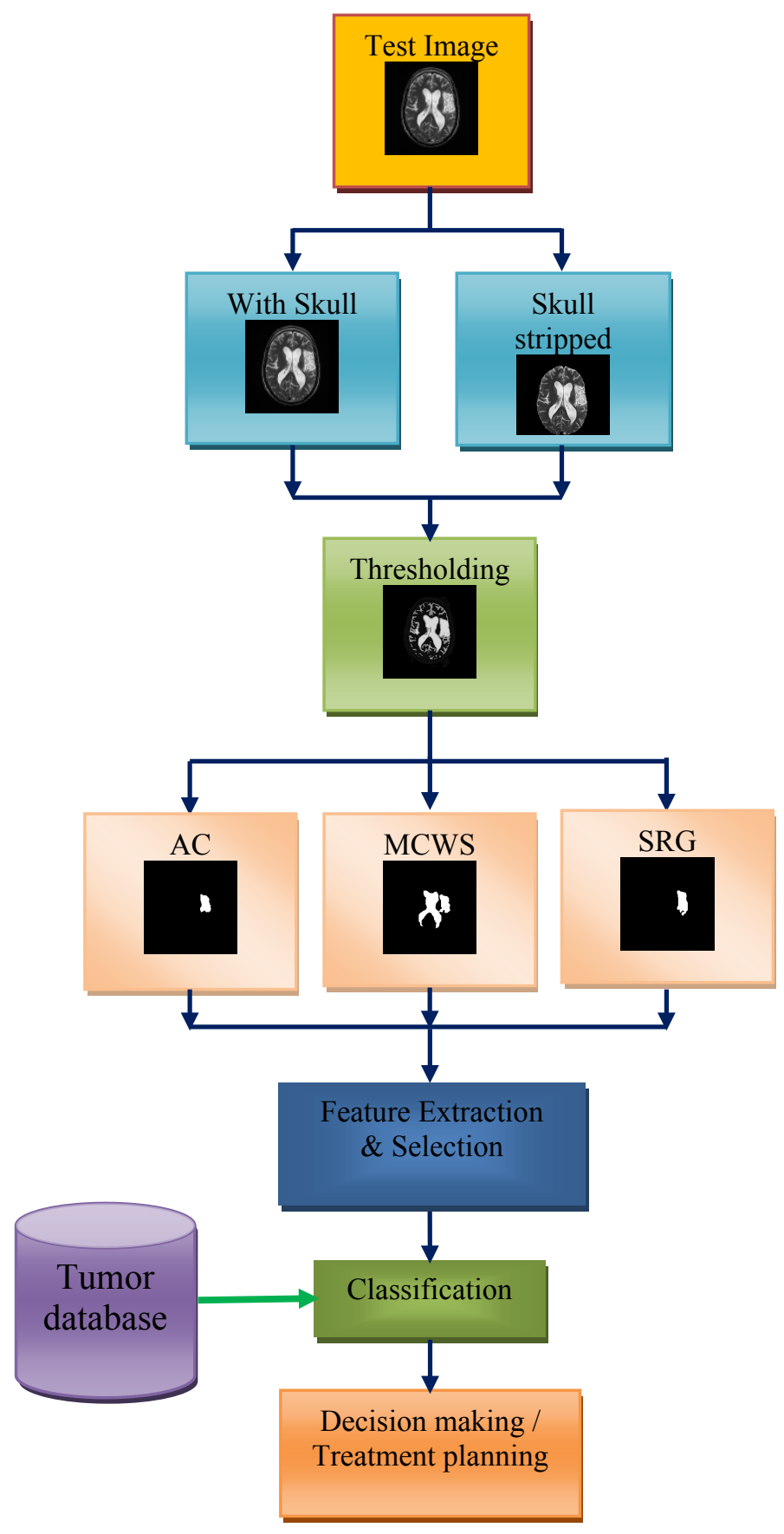

Figure 1. Overview of the proposed brain MRI examination procedure (AC- Active Contour, MCWS- Marker Controlled Watershed and SRG- Seed Region Growing)

Figure 1 presents the various stages involved in the proposed CDT. Initially, a 2D MRI slice is used for the assessment. In the literature, much effort is taken to strip the skull section from the brain MRI [33]. The skull can be retained or eliminated based on the procedure considered to develop the CDT. Initial enrichment of the raw MRI is to be carried with a suitable image pre-processing procedure and a chosen post-processing practice is to be considered to extract the tumor or stroke section from the brain MRI. Feature extraction methods are then employed to extract the significant information from the region of interest. The key features are then selected from the extracted features to train and test the classifier system. The classifier compares the available feature with respect to an 
existing tumor feature set and provides a result for the considered 2D test image. This result is then evaluated by a doctor, who takes the decision about the further treatment procedure to be considered to regulate or completely cure the brain abnormality. In any case, the developed CDT can only provide a suggestion, i.e. a pre-opinion, regarding the severity of the disease, and the doctor is the solitary person to take the decision regarding the treatment process.

The details of the steps considered in this CDT are presented below.

\subsection{Skull Region}

Generally, brain images acquired by CT/MRI include the skull section. MRI modalities like Flair, $\mathrm{T} 1, \mathrm{~T} 1 \mathrm{C}$ and $\mathrm{T} 2$ have noticeable skull section, and DW modality image has negligible skull region in axial, coronal and sagittal views of MRI. In Flair and T2 MRI modalities, the intensity of the skull segment is approximately similar to the tumor, since the image threshold levels are identical. Then, if an automated tumor extraction procedure is to be implemented in the CDT, then it is essential to eliminate the skull section from the brain MRI and the soft tissues like the gray matter, white matter, or tumor, can be exactly extracted and evaluated. Different skull removing procedures implemented by the researchers can be found in [21,31,33]. If the CDT contains a semi-automated tumor segmentation procedure, then the skull stripping procedure is not essential. Since, in these procedures, the operator will initialize the segmentation task by indicating the suitable location of the tumor in the brain MRI. Procedures like, Active-Contour (AC) [23,34-36], Seed-Region-Growing (SRG) [37, 38], Chan-Vese (CV) [8,39], and Distance-Regularised-Level-Set (DRLS) [40,41], falls in this category, which does not worry about the skull section. Hence the choice of skull stripping depends mainly on the CDT implemented to evaluate the brain abnormality.

\subsection{Pre-processing}

Image pre-processing involves in converting the input raw image into acceptable form by implementing procedures, such as de-noising, histogram equalization, adaptive thresholding, and multi-thresholding. In this work, the multi-thresholding procedure based on the Social Group Optimization (SGO) and Shannon's Entropy (SE) was implemented. Recent works in the literature confirm that heuristic algorithm supported tri-level thresholding is widely adopted to pre-process grayscales and RGB medical images [1-3]. The works of Rajinikanth et al. [22] and Shree et al. [42] confirm that SE offers enhanced image quality compared to other pre-processing schemes. Previous research by Rajinikanth and Satapathy [8], Dey et al. [43] and Satapathy and Naik [44], confirms that SGO is very simple in implementation and offers better result compared to the existing heuristic approaches in the literature.

\subsection{SGO}

SGO is a heuristic approach proposed in 2016 [44, 45], which is built by mimicking the activities in human group. SGO includes two core stages, namely: (i) improving part to synchronize the locations of people based on the objective function, and (ii) acquiring part that permit the agents to determine the best potential resolution for the chosen task.

The arithmetical structure of SGO is:

Gbest $_{j}=\max \left\{f\left(X_{i}\right)\right.$ fori $\left.=1,2, \ldots N\right\}$

where $X_{i}$ represents early information of people in a group, $i=1,2,3, \ldots, N$ signifies total people in a group, and $f_{j}$ denotes fitness function. 
(i) Improving part is considered to refurbish the position of each person as:

Xnew $_{i, j}=c *$ Xold $_{i, j}+R *\left(\right.$ Gbest $_{j}-$ Xold $\left._{i, j}\right)$

where Xnew symbolizes reorganized location, Xold indicates early location, Gbest denotes universal top location, $R$ points arbitrary numeral $[0,1]$ and $c$ represents the self-introspection parameter[0,1].

In this work, in order to get better results, $\mathrm{c}$ was chosen as equal to 0.2 as specified in $[8,44,45]$.

(ii) During the acquiring part, the agents will find the global solution based on knowledge updating procedure as:

Xnew $_{i, j}=$ Xold $_{i, j}+R_{a} *\left(X_{i, j}-X_{r, j}\right)+R_{b} *\left(\right.$ Gbest $\left._{j}-X_{i, j}\right)$

where $R_{a}$ and $R_{b}$ are random numbers $[0,1]$ and $X_{r, j}$ is the arbitrarily selected location of a person in the group.

The primary algorithm constraints were defined as: $\mathrm{N}=30, c=0.2$, iteration value $=1500$ and stopping criterion =maximized Shannon's entropy.

\subsection{Shannon's Entropy}

SE was developed by Kannappan [46] and detailed in Paul and Bandyopadhyay's work [47]. Recently, SE based thresholding was considered to pre-process medical images [2,42]. In order to explain SE, let us consider an image with dimension $\mathrm{A}^{*} \mathrm{~B}$. The gray-level pixel organizations of image (h,v) is expressed as $\mathrm{G}(\mathrm{h}, \mathrm{v})$, for $h \in\{1,2, \ldots, A\} \& v \in\{1,2, \ldots, B\}$. Let $L$ be the number of gray levels of the test image and the set of all gray values $\{0,1,2, \ldots, L-1\}$ be symbolized as $Z$, in such a way that:

$$
G(h, v) \in Z \quad \forall(h, v) \in \text { image }
$$

Then, the normalized histogram will be: $X=\left\{x_{0}, x_{1}, \ldots, x_{\mathrm{L}-1}\right\}$.

For tri-level thresholding case, Eq.4 becomes:

$$
\begin{aligned}
& X(T)=x_{0}\left(t_{1}\right)+x_{l}\left(t_{2}\right)+x_{2}\left(t_{3}\right) \\
& f(T)=\max _{T}\{X(T)\}
\end{aligned}
$$

where, $\mathrm{T}=\left\{t_{1}, t_{2}, \ldots, t_{L}\right\}$ is the threshold value, $\mathrm{X}=\left\{x_{0}, x_{1}, \ldots, x_{\mathrm{L}-1}\right\}$ is the normalized histogram, and $f(T)$ is the optimal threshold. Other details on SE can be found in [2].

\subsection{Post-processing}

Post-processing scheme is considered to extract the Region-Of-Interest (ROI) from the preprocessed test image. Irrespective of MRI's modality, this scheme can segment the stroke or tumor section, i.e. the ROI, with greater accuracy. Based on the requirement, automated as well as semiautomated ROI mining procedures can be implemented. The work of Rajinikanth and Satapathy [8] presents a comprehensive assessment of various segmentation procedures, tested and verified on the brain stroke MRI dataset called ISLES2015.

In this work, commonly used ROI mining procedures, such as AC [30], MCWS [48] and SRG [37] approaches were implemented to extract the abnormal section from brain MRI of Flair/DW modality.

AC implements a bounding box around the tumor section to be extracted. It operates based on an adjustable snake, which continuously groups the related pixel values lies within the bounding box and 
converges towards the centre of the ROI based on the iteration value. More information on the AC implemented can be found in $[35,36]$.

MCWS is also a famous procedure used to extract the ROI. MCWS combines the benefit of Sobel's edge detection, marker inhibited morphological function, and segmentation. This algorithm identifies the whole alike pixels to mine the ROI. Detailed explanation on MCWS is available in [8].

SRG is an operator initiated segmentation approach and widely adopted to mine the ROI in medical images. It needs the initiation of a seed at a point or a pixel on the ROI. When a seed point is identified, the section will cultivate by connecting possible similar neighboring pixels available in ROI [34]. SRG is one of the widely adopted semi-automated image segmentation scheme [8].

\subsection{ROI Assessment}

The aim of this work was to extract the abnormal region from MR image and to calculate the texture features for additional examination. This work considers a benchmark ISLES and BRATS image dataset, in which test images are allied with expert's image known as Ground Truth (GT). After extracting the Region of Interest (ROI) from the MR image, a relative examination among the ROI and GT is performed and evaluation metrics, like Jaccard, Dice, False Positive Rate (FPR), and False Negative Rate (FNR), are computed [8, 33]:

$$
\begin{aligned}
& \operatorname{JSC}\left(I_{g t}, I_{t}\right)=I_{g t} \cap I_{t} / I_{g t} \cup I_{t} \\
& \operatorname{DSC}\left(I_{g t}, I_{t}\right)=2\left(I_{g t} \cap I_{t}\right) /\left|I_{g t}\right| \cup\left|I_{t}\right| \\
& \operatorname{FPR}\left(I_{g t}, I_{t}\right)=\left(I_{g t} / I_{t}\right) /\left(I_{g t} \cup I_{t}\right) \\
& \operatorname{FNR}\left(I_{g t}, I_{t}\right)=\left(I_{t} / I_{g t}\right) /\left(I_{g t} \cup I_{t}\right)
\end{aligned}
$$

where, $I_{g t}$ represents the GT and $I_{t}$ symbolizes the mined region.

Additionally, image statistical values, such as sensitivity, specificity, accuracy, precision, BCR and BER, are also computed [49,50]:

$$
\begin{aligned}
& \text { Sensitvity }=T_{P} /\left(T_{P}+F_{N}\right) \\
& \text { Specificity }=T_{N} /\left(T_{N}+F_{P}\right) \\
& \text { Accuracy }=\left(T_{P}+T_{N}\right) /\left(T_{P}+T_{N}+F_{P}+F_{N}\right) \\
& \text { Precision }=T_{P} /\left(T_{P}+F_{P}\right) \\
& B C R=1 / 2\left(T_{P} /\left(T_{P}+F_{N}\right)+T_{N} /\left(T_{N}+F_{P}\right)\right) \\
& B E R=1-B C R
\end{aligned}
$$

where $I_{G T}$ is GT, $I_{S}$ is the ROI, $T_{N}, T_{P}, F_{N}$ and $F_{P}$ are the related measures [51-54].

\subsection{Feature Extraction and Selection}

The texture description of the ROI is extracted with the Haralick scheme that is extensively recognized as the Gray Level Co-occurrence Matrix (GLCM) [55-57]. The earlier research works with GLCM confirm that texture descriptions are usually adopted to collect essential information from the ROI. These features are then considered to train, test and validate the classifier units, which is created to categorize the brain tumor under analysis into benign and malignant, which may assist the doctor in taking the right decision and also to identify appropriate treatment method to cure or regulate the brain abnormality.

In this work, the GLCM is considered to extract around 20 texture features from the ROI. After extracting the features, it is essential to select the key features to minimize the complexity in building the disease classifier units. In the literature, proper guidelines are proposed to select the main features 
of the GLCM, like in [58-62]. Hence, in this work, the features, such as contrast, correlation, clusterprominence, energy, entropy, sum-entropy, IMC1 and IMC2, are considered to train the classifier.

\subsection{Classification}

Classifiers are employed to segregate the available data into various classes based on requirement. In the literature, a substantial number of classifiers are available [61-63]. During the brain tumor assessment task, it is essential to take complete care while choosing the slice's ROI features to train and test the classifier. Usually, the MRI exam is available as a 3D image, however, during the evaluation process, various $2 \mathrm{D}$ slices are extracted to minimize the complexity in examination. In this process, one can note that, while converting the 3D image into 2D slices, the tumor section will not be same in the every slice. Some slice may contain the information of edema and some other may show the tumor core. If entire slice of the 2D MRI of a single patient is considered during the training and testing phase of the classifier, the final classification result will be wrong and that will affect the decision making or treatment planning process. Hence, in order to get a fair and accurate classification, it is essential to consider the 2D slice which contains the maximal tumor size, i.e. edema plus tumor core.

Further, it is essential to consider similar size of 2D MRI slices to attain better classification outcome. In this work, three classes of MRI datasets, such as ISLES (256x256 pixels), BRATS (236x216 pixels) and clinical image (256x256 pixels), were considered. In order to train and test the classifier system, the ISLES dataset (38 patient's images) was considered. From this dataset, 32 patient's 2D slices were considered to train the classifier and the 6 patient's data was considered to test the accuracy and the performance of the classifier.

In this work, Adaptive Neuro Fuzzy Inference System (ANFIS) based classifier system was implemented. ANFIS is a familiar classifier system built based on the neural network principle and Takagi-Sugeno fuzzy inference configuration. The work of Manickavasagam et al. [60] verified that ANFIS has the potential of presenting the best classification outcome compared to the Neural Network supported classifiers due to its hybrid nature.

The inference structure in ANFIS works is based on a set of fuzzy IF-THEN rules [64-66], which have learning prospective to estimate nonlinear functions. Due to its competence, ANFIS is generally used by the researchers to classify signals and images.

The ANFIS classifier is trained as follows:

The training session is realized in two trails, such as forward and reverse. The Haralick's features considered (S) are divided into two sets:

$S=S_{1} \oplus S_{2}$

where $S$ denotes the total parameters, $S_{1}$ represents the principle values, $S_{2}$ signifies the consequent values, and $\oplus$ represents the direct sum.

In forward training session, least square approach is applied, and during the backward training session, minimization of the overall quadratic cost value is adopted. While executing these procedures, the training algorithm determines the optimal set of parameters, which can be used to assist the testing process. The initial parameters for the ANFIS classifier were assigned as follows: initially, generalised bell-shaped membership function was chosen for the fuzzy system, iteration number was defined as 250 , parameter adaptation step size was chosen as 0.01 and the error acceptance was fixed as 0.005 . 


\section{Results and Discussions}

This section offers the results attained with the proposed CDT. The literature confirms the availability of a significant number of image processing procedures for MRI examination. The proposed work implements a two-stage procedure to examine the benchmark brain MRI and the MRI images obtained from the clinic. This work considers the assistance of the modern heuristic procedure called the SGO along with the SE. A detailed assessment among the existing segmentation procedures, such as AC, MCWS and SRG, was also conducted. Finally, a tumor classification system was implemented based on the ANFIS, and trained and tested using the well-known texture features called GLCM. The developed CDT was executed in a workstation with an AMD C70 Dual Core $1 \mathrm{GHz}$ CPU with $4 \mathrm{~GB}$ of RAM and implemented in Matlab.

Initially, the ISLES database of 38 volunteers was considered for the evaluation. This dataset contains the MRI acquired with different modalities, such as Flair, T1 and DW, with a pixel dimension of $77 \times 77$. This dataset also includes ground truths offered by an expert. It is a 3D dataset and supports the axial, coronal and sagittal views. Initially, the chosen $2 \mathrm{D}$ slices from the $3 \mathrm{D}$ images were extracted and then re-sized into 256x256 pixel image. During this study, only the axial view images were considered for the evaluation $[8,67,68]$.

Table 1. Sample test images of the ISLES dataset with Flair and DW modalities

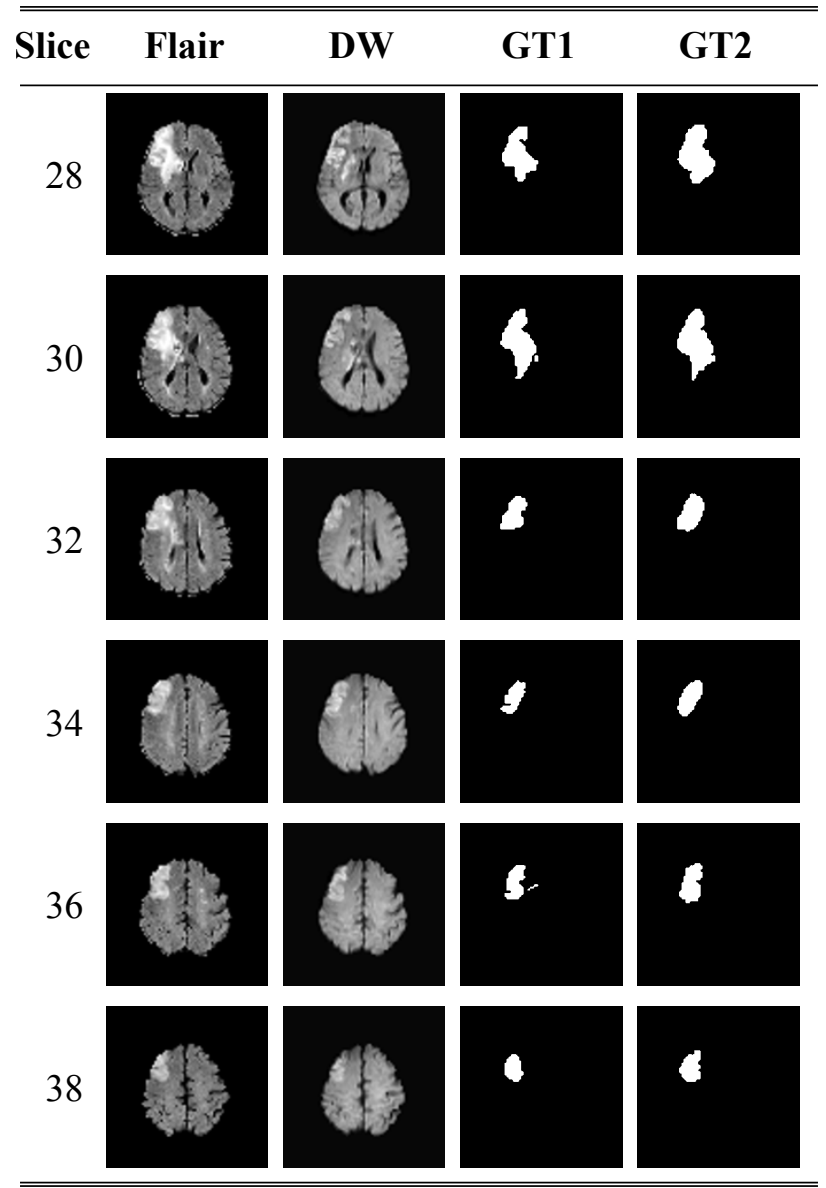

Table 1presents the chosen test images (6 slices) and the corresponding ground truths (GT1 \& GT2). These images are free from the skull section, hence it can be considered the implementation of semi-automated as well as automated segmentation procedures. Initially, 2D slices of Flair modality (slice number from 28 to 38 ) were considered for the evaluation and the results depicted in Table 2 were obtained. Firstly, a tri-level thresholding was implemented based on the SGO+SE and the 
corresponding outcome was registered in the table. Later, the segmentation procedure (AC/MCWS/SRG) was implemented to extract the abnormal section from the pre-processed image and the corresponding outcomes were registered. Therefore, Table 2 presents the results of the preprocessing step, post-processing schemes and the extracted brain abnormality. Similar procedure was repeated for the 2D slices of the DW modality and the corresponding results were registered. From this experiment, it was found that the extracted ROI of flair and DW were with $95 \%$ identical, In order to assess the performance of the implemented segmentation methods, like AC, MCWS and SRG, a comparative analysis was executed between the ROI and the GT. Initially, the ROI was compared against GT1, and later against the GT2, and the corresponding outcomes were registered, Tables 3 and 4. In both the tables, the JI, DC, ACC, PRE, SEN, SPE, BCR and BER are indicated in percentage (\%), and the average values are denoted as Avg1, Avg2, and Avg3.

Table 2. Pre-processing and Post-processing results obtained for the sample images of the ISLES

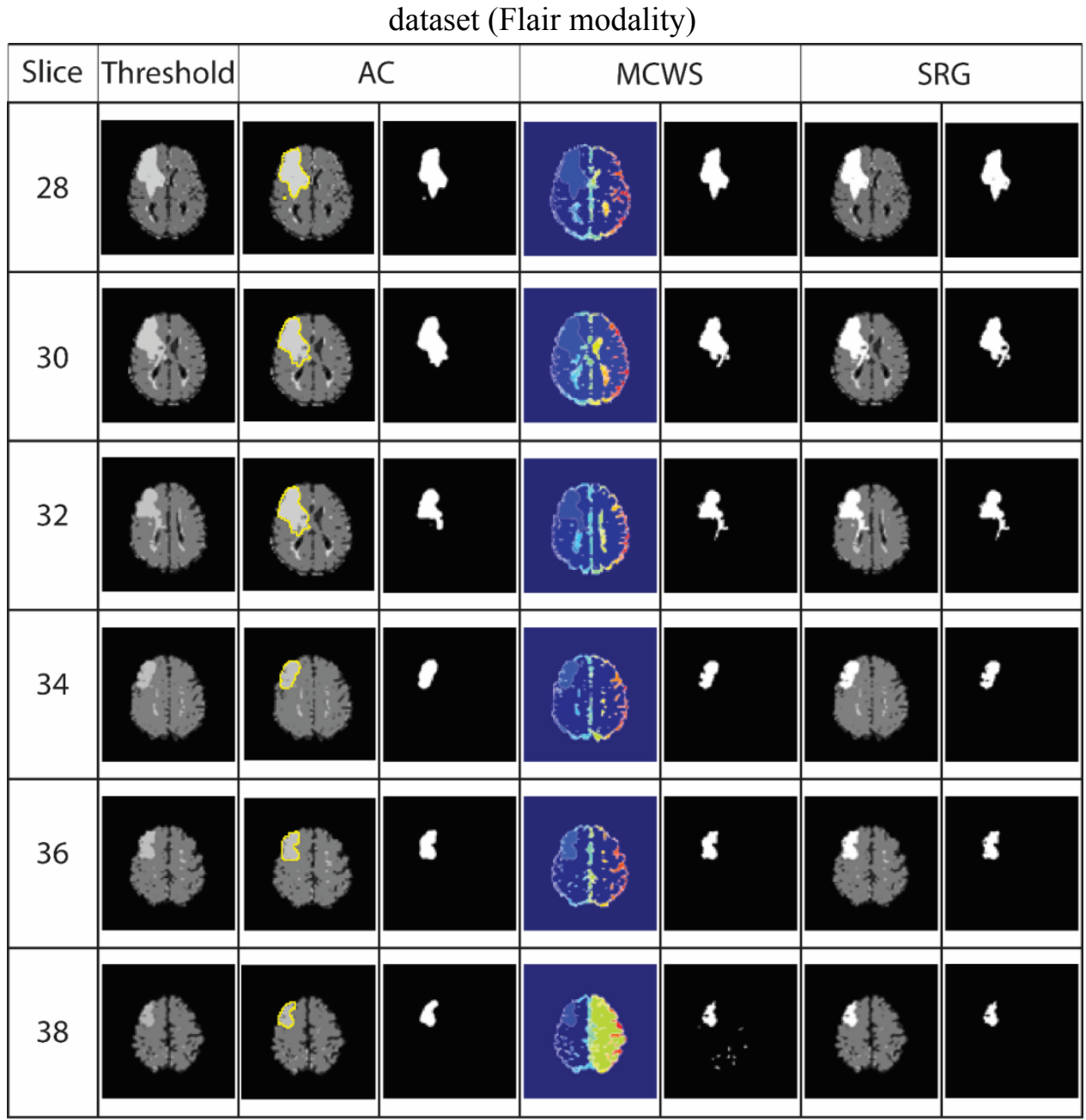


Table 3. Image similarity measures obtained between the extracted ROI and related GT1

\begin{tabular}{|c|c|c|c|c|c|c|c|}
\hline & Slice & TPrate & FNrate & TNrate & FPrate & JI & DC \\
\hline \multirow{7}{*}{$\stackrel{U}{\&}$} & 28 & 0.8068 & 0.1932 & 0.9997 & 0.0003 & 80.19 & 89.01 \\
\hline & 30 & 0.8689 & 0.1311 & 0.9962 & 0.0038 & 81.27 & 89.67 \\
\hline & 32 & 0.6349 & 0.3651 & 0.9998 & 0.0002 & 63.19 & 77.44 \\
\hline & 34 & 0.7480 & 0.2520 & 0.9997 & 0.0003 & 73.95 & 85.02 \\
\hline & 36 & 0.7920 & 0.2080 & 0.9995 & 0.0005 & 77.68 & 87.44 \\
\hline & 38 & 0.7702 & 0.2298 & 0.9988 & 0.0012 & 72.20 & 83.86 \\
\hline & Avg1 & 0.7701 & 0.2299 & 0.9989 & 0.0011 & 74.75 & 84.41 \\
\hline \multirow{7}{*}{$\frac{\pi}{3}$} & 28 & 0.8793 & 0.1207 & 0.9990 & 0.0010 & 86.11 & 92.54 \\
\hline & 30 & 0.9453 & 0.0547 & 0.9921 & 0.0079 & 80.63 & 89.28 \\
\hline & 32 & 0.6719 & 0.3281 & 0.9992 & 0.0008 & 65.63 & 79.25 \\
\hline & 34 & 0.8650 & 0.1350 & 0.9991 & 0.0009 & 82.67 & 90.51 \\
\hline & 36 & 0.9109 & 0.0891 & 0.9985 & 0.0015 & 84.63 & 91.67 \\
\hline & 38 & 0.6492 & 0.3508 & 0.9936 & 0.0064 & 67.18 & 73.72 \\
\hline & Avg2 & 0.8203 & 0.1797 & 0.9969 & 0.0031 & 77.81 & 86.16 \\
\hline \multirow{7}{*}{\begin{tabular}{l}
0 \\
\multirow{2}{\infty}{}
\end{tabular}} & 28 & 0.8789 & 0.1211 & 0.9989 & 0.0011 & 85.81 & 92.36 \\
\hline & 30 & 0.9440 & 0.0560 & 0.9911 & 0.0089 & 78.60 & 88.02 \\
\hline & 32 & 0.6719 & 0.3281 & 0.9992 & 0.0008 & 65.63 & 79.25 \\
\hline & 34 & 0.8709 & 0.1291 & 0.9989 & 0.0011 & 82.38 & 90.34 \\
\hline & 36 & 0.9149 & 0.0851 & 0.9983 & 0.0017 & 83.88 & 91.23 \\
\hline & 38 & 0.8938 & 0.1062 & 0.9962 & 0.0038 & 68.11 & 81.03 \\
\hline & Avg3 & 0.8624 & 0.1376 & 0.9971 & 0.0029 & 77.40 & 87.04 \\
\hline
\end{tabular}

Table 4. Image statistical measures obtained between the extracted ROI and related GT1

\begin{tabular}{cccccccc}
\hline \hline & Slice & ACC & PRE & SEN & SPE & BCR & BER \\
\hline & 28 & 99.10 & 99.97 & 98.96 & 99.24 & 99.10 & 0.89 \\
30 & 95.92 & 99.62 & 99.28 & 92.67 & 95.98 & 4.02 \\
& 32 & 98.95 & 99.98 & 98.66 & 99.25 & 98.95 & 1.05 \\
\multirow{4}{*}{34} & 98.95 & 99.97 & 99.42 & 98.48 & 98.95 & 1.05 \\
& 36 & 96.35 & 99.86 & 99.50 & 93.30 & 96.40 & 3.60 \\
& 38 & 95.74 & 99.88 & 99.59 & 92.03 & 95.81 & 4.19 \\
$\mathbf{Z}$ & Avg1 & $\mathbf{9 7 . 5 0}$ & $\mathbf{9 9 . 8 8}$ & $\mathbf{9 9 . 2 3}$ & $\mathbf{9 5 . 8 3}$ & $\mathbf{9 7 . 5 3}$ & $\mathbf{2 . 4 7}$ \\
& 28 & 98.54 & 99.91 & 99.43 & 97.67 & 98.55 & 1.45 \\
& 30 & 91.90 & 99.20 & 99.75 & 84.67 & 92.21 & 7.79 \\
32 & 97.75 & 99.92 & 98.92 & 96.59 & 97.75 & 2.25 \\
& 34 & 97.30 & 99.91 & 99.74 & 94.91 & 97.33 & 2.67
\end{tabular}




\begin{tabular}{|c|c|c|c|c|c|c|c|}
\hline & 36 & 93.84 & 99.76 & 99.83 & 88.21 & 94.02 & 5.98 \\
\hline & 38 & 85.92 & 99.62 & 99.62 & 74.11 & 86.87 & 13.13 \\
\hline \multirow{8}{*}{ 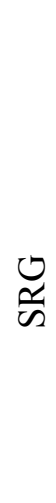 } & Avg2 & 94.21 & 99.72 & 99.55 & 89.36 & 94.45 & 5.54 \\
\hline & 28 & 98.37 & 99.89 & 99.44 & 97.32 & 98.38 & 1.62 \\
\hline & 30 & 90.75 & 99.09 & 99.75 & 82.57 & 91.16 & 8.84 \\
\hline & 32 & 97.74 & 99.92 & 98.92 & 96.59 & 97.75 & 2.25 \\
\hline & 34 & 96.76 & 99.89 & 99.76 & 93.84 & 96.80 & 3.20 \\
\hline & 36 & 93.19 & 99.74 & 99.84 & 86.98 & 93.41 & 6.59 \\
\hline & 38 & 86.03 & 99.62 & 99.87 & 74.11 & 86.99 & 13.01 \\
\hline & Avg3 & 93.81 & 99.69 & 99.59 & 88.57 & 94.08 & 5.92 \\
\hline
\end{tabular}

An analysis between the extracted ROI and related GT1 was performed to assess the performance of the considered segmentation procedures. The JI offered by the MCWS was superior to AC and SRG, also the DC of SRG was better than the alternatives. The values in Table 4 confirm that the statistical values of the AC were better compared to MCWS and SRG approaches. This confirms that AC can be considered to examine 2D MR images with superior accuracy. Figure 2 depicts the pictorial demonstration of the image similarity and statistical measures. This confirms that, for most of the cases, the AC offers better result.

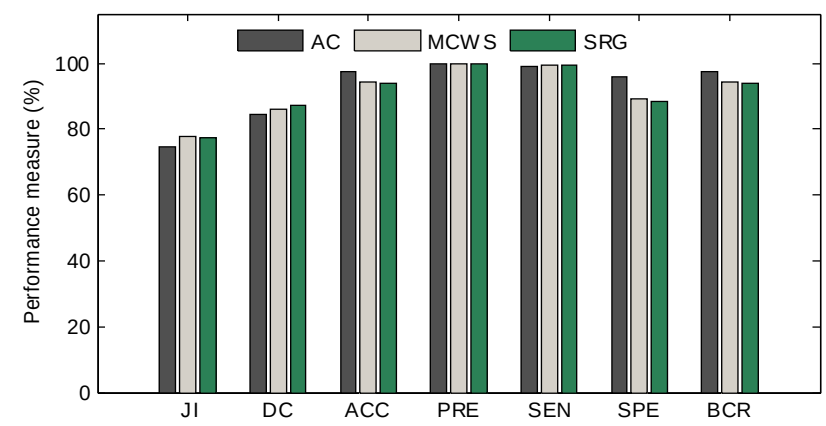

Figure2. Graphical assessment for the image similarity and statistical measures of Flair modality MRI slices of the ISLES dataset

The examination procedure implemented for the ISLES dataset was then repeated on the BRATS database. During this investigation, the 2D slices of flair modality were chosen for the study (slices number 100 to 140). Table 5 presents the chosen test images and the corresponding GT. The GT section includes the edema and the tumor core. Hence, the extraction of the ROI was the challenging task in these images, since the edema pixel contrast is similar to the normal pixel value of the brain tissues. As discussed earlier, the pre-processing and the post-processing sections were implemented for the considered test images and the related outcomes were assessed, Table 5. A comparative analysis among the extracted ROIs and related GTs was then conducted and the corresponding image similarity and statistical values computed and registered, Table 6. Figure 3 presents the pictorial representation of the performance comparisons. Table 6 and Figure 3 confirm that, for the BRATS dataset, the outcomes obtained for the AC were superior to MCWS and SRG. This confirms that, in this CDT, the AC based segmentation offers better results; however, the average CPU time of CDT with AC is $72.18 \mathrm{sec}$, whereas CDT with MCWS offers the result in $46.93 \mathrm{sec}$ and with SRG in 52.60sec. 
Table 5. Sample test images of the BRATS (Flair modality)

\begin{tabular}{|c|c|c|c|c|c|}
\hline & S100 & S110 & S120 & S130 & S140 \\
\hline Flair & & & & & \\
\hline GT & 3 & & 3 & & \\
\hline $\begin{array}{c}\text { Threshold } \\
\text { image }\end{array}$ & & & & & \\
\hline $\mathrm{AC}$ & & & & & \\
\hline MCWS & & & & & \\
\hline SRG & & & & & \\
\hline
\end{tabular}

Table 6. Performance measures obtained for the sample BRATS images

\begin{tabular}{ccccccccc}
\hline \hline Method & JI & \multirow{2}{*}{ DC } & ACC & PRE & SEN & SPE & BCR & BER \\
\hline AC & $\mathbf{8 2 . 1 6}$ & $\mathbf{8 8 . 5 2}$ & $\mathbf{9 6 . 7 3}$ & 99.48 & 99.74 & $\mathbf{9 4 . 2 9}$ & 95.18 & $\mathbf{2 . 7 3}$ \\
MCWS & 81.94 & 88.16 & 96.17 & $\mathbf{9 9 . 6 6}$ & 99.59 & 93.95 & $\mathbf{9 6 . 2 4}$ & 3.28 \\
SRG & 82.04 & 87.84 & 96.58 & 99.40 & $\mathbf{9 9 . 7 6}$ & 94.06 & 94.86 & 3.56 \\
\hline \hline
\end{tabular}

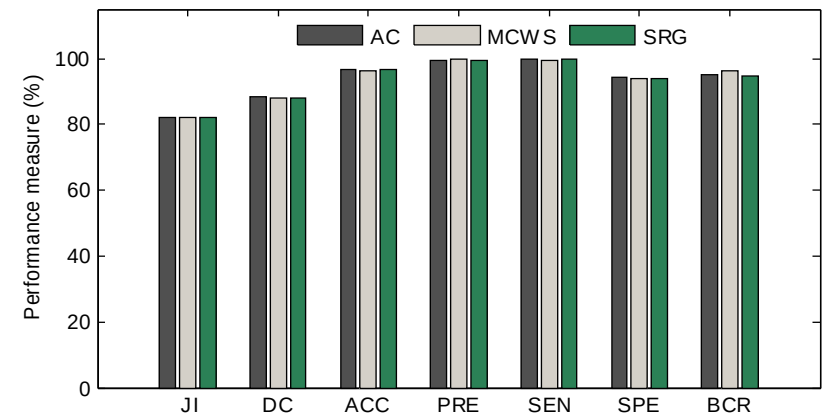

Figure 3. Graphical appraisal for similarity and statistical measures of Flair modality MRI slices of the BRATS dataset

Furthermore, the proposed CDT was tested on real clinical images of brain MRI acquired with DW and Flair modalities in Proscans laboratory [69]. Initially, the original images were down scaled to $256 \times 256$ pixels and then the proposed CDT was used to extract and examine the tumor section from the 2D brain slices. Figure 4 presents the 2D slices of the DW modality MRI with the slices number from SDW27 to SDW36. 

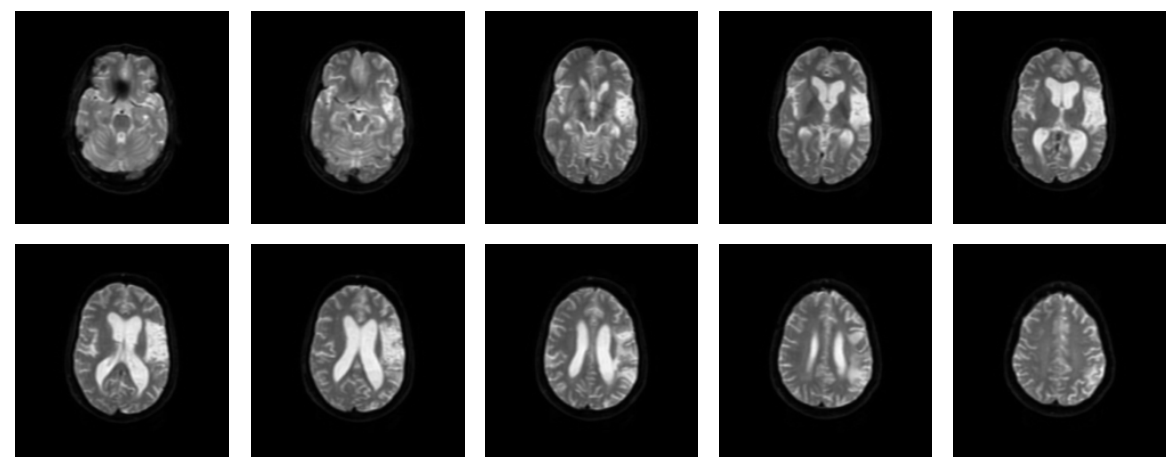

Figure 4. 2D slices of the brain MRI acquired with the DW modality

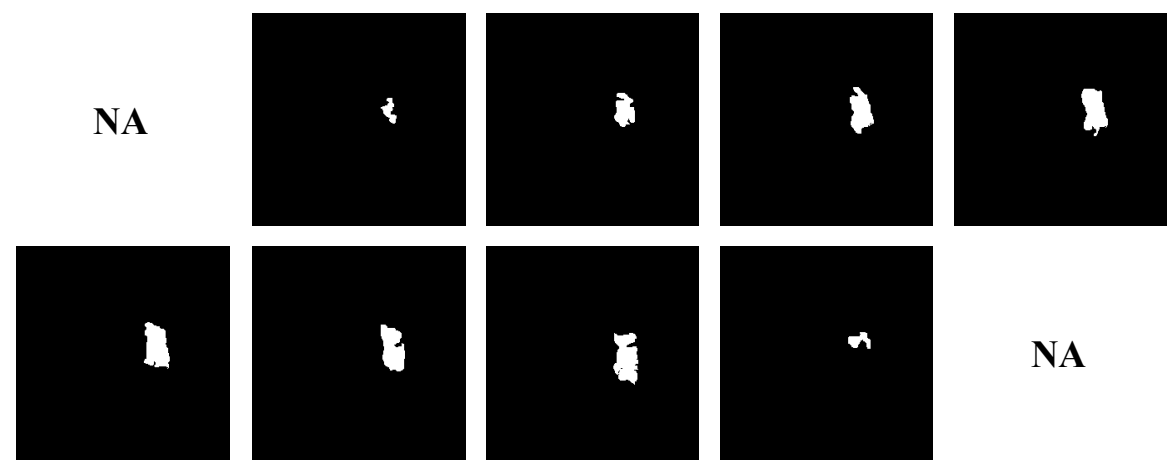

Figure 5. Outcome of the proposed CDT with AC for the chosen slices of the DW MRI modality

Table 7. Extracted GLCM features for the DW modality images presented in Figure 5

\begin{tabular}{ccccccccc}
\hline \hline Measures & SDW28 & SDW29 & SDW30 & SDW31 & SDW32 & SDW33 & SDW34 & SDW35 \\
\hline Autocorrelation & 1.0118 & 1.0318 & 1.0464 & 1.0372 & 1.0415 & 1.0418 & 1.0599 & 1.0138 \\
Contrast & 0.0019 & 0.0026 & 0.0024 & 0.0026 & 0.0038 & 0.0036 & 0.0042 & 0.0017 \\
Correlation & 0.8078 & 0.8828 & 0.9232 & 0.9142 & 0.9163 & 0.9048 & 0.9115 & 0.8256 \\
$\begin{array}{c}\text { Cluster- } \\
\text { prominence }\end{array}$ & 0.0558 & 0.1513 & 0.2223 & 0.2281 & 0.2374 & 0.2506 & 0.2797 & 0.0648 \\
Cluster-shade & 0.0288 & 0.0777 & 0.1140 & 0.1174 & 0.1287 & 0.1294 & 0.1443 & 0.0334 \\
Dissimilarity & 0.0019 & 0.0026 & 0.0024 & 0.0024 & 0.0029 & 0.0031 & 0.0036 & 0.0017 \\
Energy & 0.9899 & 0.9757 & 0.9664 & 0.9518 & 0.9476 & 0.9530 & 0.9561 & 0.9886 \\
Entropy & 0.0362 & 0.0743 & 0.0950 & 0.1074 & 0.1163 & 0.2195 & 0.1222 & 0.0399 \\
Homogeneity & 0.9992 & 0.9987 & 0.9988 & 0.9927 & 0.9983 & 0.9979 & 0.9982 & 0.9992 \\
Max- & 0.9949 & 0.9877 & 0.9829 & 0.9793 & 0.9716 & 0.9756 & 0.9776 & 0.9943 \\
probability & & & & & & & & \\
Sum-average & 2.0085 & 2.0220 & 2.0317 & 2.0157 & 2.0175 & 2.0261 & 2.0412 & 2.0098 \\
Sum-variance & 3.9099 & 3.8415 & 3.8175 & 3.6118 & 3.7108 & 3.7163 & 3.7798 & 3.9027 \\
Sum-entropy & 0.0351 & 0.0725 & 0.0933 & 0.0982 & 0.1084 & 0.1102 & 0.1169 & 0.0387 \\
Difference- & 0.0016 & 0.0026 & 0.0024 & 0.0027 & 0.0030 & 0.0031 & 0.0036 & 0.0017
\end{tabular}


variance

\begin{tabular}{ccccccccc}
$\begin{array}{c}\text { Difference- } \\
\text { entropy }\end{array}$ & 0.0138 & 0.0178 & 0.0169 & 0.0182 & 0.0198 & 0.0206 & 0.0237 & 0.0125 \\
IMC1 & -0.6841 & -0.7736 & -0.8344 & -0.7806 & -0.7927 & -0.7994 & -0.8095 & -0.7055 \\
IMC2 & 0.1922 & 0.2992 & 0.3566 & 0.3618 & 0.3715 & 0.3774 & 0.3871 & 0.2063 \\
ID & 0.0000 & 0.0000 & 0.0000 & 0.0000 & 0.0000 & 0.0000 & 0.0000 & 0.0000 \\
IDN & 0.9995 & 0.9991 & 0.9992 & 0.9982 & 0.9993 & 0.9991 & 0.9988 & 0.9994 \\
IDMN & 0.9997 & 0.9995 & 0.9995 & 0.9993 & 0.9996 & 0.9991 & 0.9993 & 0.9997 \\
\hline
\end{tabular}

The proposed CDT was implemented with the AC segmentation, Figure 5 confirms that the tumor section in SDW27 and SDW36 is less visible compared to other slices. After extracting the possible tumor section (ROI) from the chosen 2D MRI slices, the essential texture features were then extracted and registered, Table 7. Table 7 presents the 20 features obtained using the GLCM, in which few chosen features have vital information regarding the tumor section, which further can be considered to train and test the ANFIS classifier system developed in this work.

Similar procedure was considered for the 2D flair MRI modality shown in Figure 6, Figure 7. From these figures, one can observe that the proposed CDT can assist the extraction of the timorous section from the real clinical brain Flair/DW MRI modalities. Hence, in future, this CDT can be considered as a diagnosing and assisting tool for doctors to take the appropriate decision regarding the tumor section.
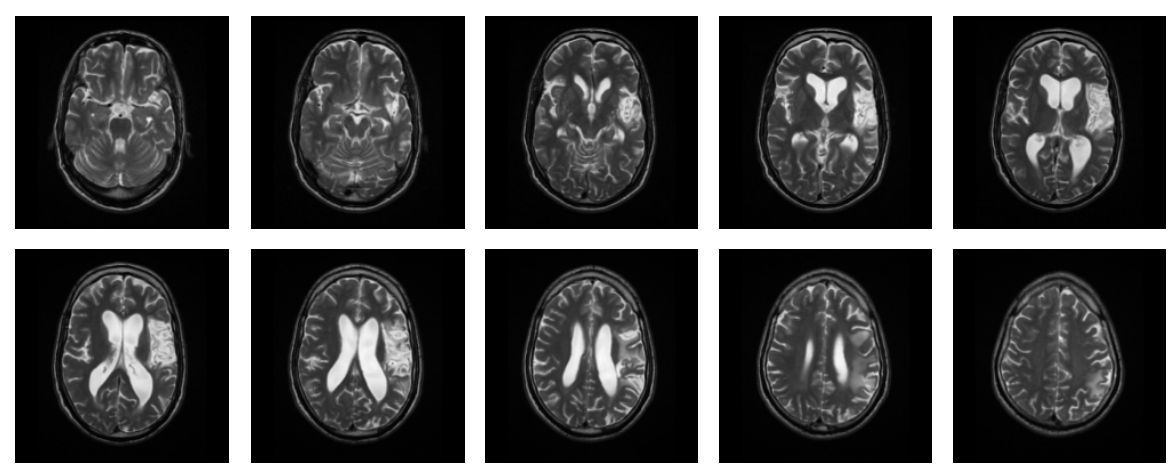

Figure 6. 2D slices of the brain MRI acquired with Flair modality

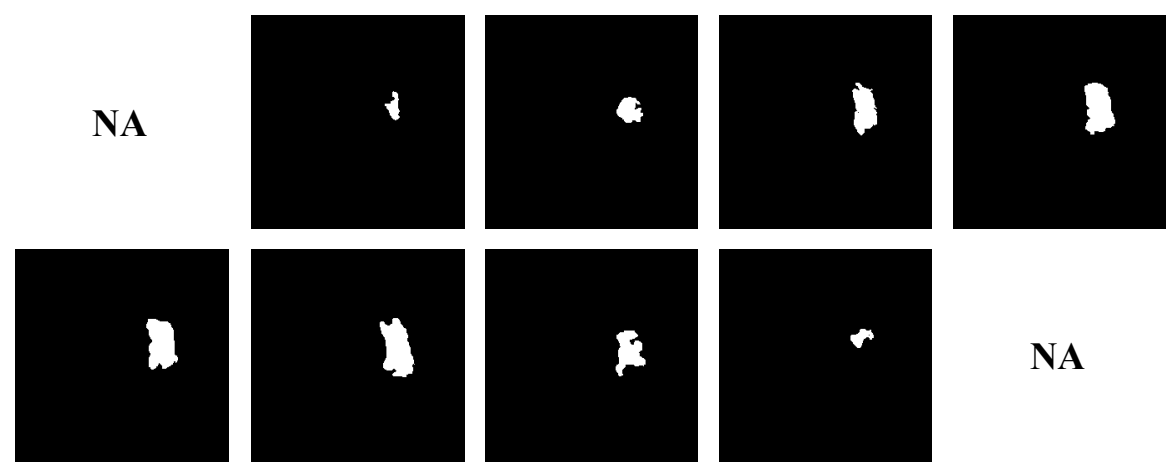

Figure 7. Results of proposed CDT with AC for chosen slices of Flair MRI modality 
Finally, the extracted and selected features were then considered to train and test the ANFIS classifier system. The image size of the ISLES and the real clinical images were identical $(256 \times 256$ pixels). Hence, the extracted GLCM features of 32 images of ISLES were considered to train the classifier system and the remaining 6 images were considered to test the ANFIS classifier. This test offered an average accuracy of $94.51 \%$, which confirmed that the proposed classifier system is efficient. In future, this ANFIS classifier system can be trained using a large number of 2D brain MRI slices and also it can be validated against the other existing classifiers in the literature.

\section{Conclusion}

This article proposed a Computerized Disease inspection Tool (CDT) using a Hybrid Image Processing (HIP) procedure. The implemented HIP technique implements the combination of the SGO assisted Shannon's tri-level thresholding and segmentation based on AC/MCWS/SRG procedures. During the experimental evaluation, the benchmark ISLES 2015 and BRATS datasets were considered for the initial assessment. Later, the proposed CDT was tested on real time tumor MRI of Flair and DW modalities obtained from a medical center. Finally, the GLCM features for the tumor section were extracted, and an ANFIS classifier was developed based on the available GLCM features. 32 patient's tumor features were used to train the ANFIS classifier and 6 patient's feature sets considered for the testing phase. For the used clinical dataset, the ANFIS classifier offered a classification accuracy of $94.51 \%$. In future, the ANFIS classifier can be validated against the classifiers existing in the literature.

\section{Compliance with Ethical Standards}

\section{Ethical Approval}

All procedures performed in studies involving human participants were in accordance with the ethical standards of the institutional and/or national research committee and with the 1964 Helsinki declaration and its later amendments or comparable ethical standards.

Conflict of interest: The authors declare that they have no conflict of interest

Informed Consent: Informed consent was obtained from all individual participants included in the study.

\section{References}

1. Dey N, Ashour AS, Beagum S, Pistola DS, Gospodinov M, Gospodinova EP, Tavares JMRS (2015) Parameter optimization for local polynomial approximation based intersection confidence interval filter using genetic algorithm: an application for brain MRI image de-noising. J Imaging 1(1):60-84. https://doi.org/10.3390/jimaging1010060

2. Rajinikanth V, Satapathy SC, Fernandes SL, Nachiappan S (2017) Entropy based segmentation of tumour from brain MR images-a study with teaching learning based optimization. Pattern Recogn Lett 94: 87-94. https://doi.org/10.1016/j.patrec.2017.05.028

3. Rajinikanth V, Fernandes SL, Bhushan B, Sunder NR (2018) Segmentation and analysis of brain tumour using Tsallis entropy and regularised level set. LNEE 434:313-321. https://doi.org/10.1007/978-981-10-4280-5_33

4. Thanaraj P, Parvathavarthini B (2017) Multichannel interictal spike activity detection using time-frequency entropy measure. Australas Phys Eng S 40(2):413-425. https://doi.org/10.1007/s13246-017-0550-6 
5. Kanmani P, Marikkannu P (2018) MRI Brain Images Classification: A multi-level threshold based region optimization technique. J Med Syst 42(4):62. https://doi.org/10.1007/s10916018-0915-8

6. Nilesh BB, Arun KR, Har PT (2017) Image analysis for MRI based brain tumour detection and feature extraction using biologically inspired BWT and SVM. I J Biomed Imag 2017(Article ID 9749108):12. https://doi.org/10.1155/2017/9749108

7. Marzena B, Khalid S (2011) Segmentation of MRI brain images for automatic detection and precise localization of tumour. AISC 102:333-341. https://doi.org/10.1007/978-3-64223154-4_37

8. Rajinikanth V, Satapathy SC (2018) Segmentation of ischemic stroke lesion in brain MRI based on social group optimization and Fuzzy-Tsallis entropy. Arab J SciEng 43(8):43654378. https://doi.org/10.1007/s13369-017-3053-6

9. Bhattacharyya D, Kim TH (2011) Brain tumour detection using MRI image analysis. CCIS 151:307-314. https://doi.org/10.1007/978-3-642-20998-7_38

10. Rajinikanth V, Raja NSM, Kamalanand K (2017) Firefly algorithm assisted segmentation of tumour from brain MRI using Tsallis function and Markov random field. Control Eng Appl Inf 19(3):97-106.

11. Isin A, Direkoglu C, Sah M (2016) Review of MRI-based brain tumour image segmentation using deep learning methods. Procedia Computer Sci 102:317-324. https://doi.org/10.1016/j.procs.2016.09.407

12. Usman K, Rajpoot K (2017) Brain tumour classification from multi-modality MRI using wavelets and machine learning. Pattern Anal Applic 20(3):871-881. https://doi.org/10.1007/s10044-017-0597-8

13. Maier O, Schröder C, Forkert ND, Martinetz T, Handels H (2015) Classifiers for ischemic stroke lesion segmentation: A comparison study. PLoS ONE 10(12):e0145118. https://doi.org/10.1371/journal.pone.0145118

14. Maier et al. (2017) ISLES 2015 - A public evaluation benchmark for ischemic stroke lesion segmentation from multispectral MRI. Med Image Anal 35:250-269. https://doi.org/10.1016/j.media.2016.07.009

15. ISLES 2015 (www.isles-challenge.org)

16. Menze et al. (2015) The multimodal brain tumour image segmentation benchmark (BRATS). IEEE T Med Imag 34(10):1993-2024. https://doi.org/10.1109/TMI.2014.2377694

17. Brain Tumour Database (BraTS-MICCAI), http://hal.inria.fr/hal-00935640.

18. Jiang et al. (2013) 3D brain tumour segmentation in multimodal MR images based on learning population-and patient-specific feature sets. Comput Med Imag Grap 37(7-8):512521. https://doi.org/10.1016/j.compmedimag.2013.05.007

19. Song T, Angelini E, Mensh B, Laine A (2004) Comparison study of clinical 3D MRI brain segmentation evaluation. Conf Proc IEEE Eng Med Biol Soc 3:1671-1674. https://doi.org/10.1109/IEMBS.2004.1403504

20. Maria et al. (2013) Registration of 3D fetal neurosonography and MRI. Med Image Anal 17(8):1137-1150. https://doi.org/10.1016/j.media.2013.07.004

21. Roopini IT, Vasanthi M, Rajinikanth V, Rekha M, Sangeetha M (2018) Segmentation of tumour from brain MRI using fuzzy entropy and distance regularised level set. LNEE 490: 297-304. https://doi.org/10.1007/978-981-10-8354-9_27

22. Rajinikanth V, Satapathy SC, Dey N, Vijayarajan R (2018) DWT-PCA Image fusion technique to improve segmentation accuracy in brain tumour analysis. LNEE 471:453-462. https://doi.org/10.1007/978-981-10-7329-8_46 
23. Rajinikanth V, Dey N, Satapathy SC, Ashour AS (2018) An approach to examine magnetic resonance angiography based on Tsallis entropy and deformable snake model. Future Gener Comp Sy 85:160-172. https://doi.org/10.1016/j.future.2018.03.025

24. Savareh BA, Emani H, Hajiabadi M, Ghafoori M (2018) Emergence of convolutional neural network in future medicine: Why and How. A review on brain tumour segmentation. P J Med Phy Engg 24(1). https://doi.org/10.2478/pjmpe-2018-0007

25. Ventura SR, Freitas DR, Tavares JMRS (2009) Application of MRI and biomedical engineering in speech production study. Comput Methods Biomech Biomed Engin 12(6):671-681. https://doi.org/10.1080/10255840902865633

26. Maier O, Wilms M, Gablentz VDJ, Krämer UM, Münte TF, Handels H (2015) Extra tree forests for sub-acute ischemic stroke lesion segmentation in MR sequences. J Neurosci Methods 240:89-100. https://doi.org/10.1016/j.jneumeth.2014.11.011

27. Mitra et al. (2014) Lesion segmentation from multimodal MRI using random forest following ischemic stroke. NeuroImage 98:324-335. https://doi.org/10.1016/j.neuroimage.2014.04.056

28. Tian Z, Dey N, Ashour AS, McCauley P, Shi F (2017) Morphological segmenting and neighborhood pixel-based locality preserving projection on brain fMRI dataset for semantic feature extraction: an affective computing study. Neural Comput Appl 1-16. https://doi.org/10.1007/s00521-017-2955-2

29. Bibicu D, Moraru L, Biswas A (2013) Thyroid nodule recognition based on feature selection and pixel classification methods. J Digit Imaging 26(1):119-128. https://doi.org/10.1007/s10278-012-9475-5.

30. Punga MV, Gaurav R, Moraru L (2014) Level set method coupled with energy image features for brain MR image segmentation. Biomed Tech (Berl) 59(3):219-229. https://doi.org/10.1515/bmt-2013-0111

31. Moldovanu S, Moraru L, Biswas A (2015) Robust skull stripping segmentation based on irrational mask for magnetic resonance brain images. J Digit Imaging 28(6):738-747. https://doi.org/10.1007/s10278-015-9776-6

32. Moraru L, Moldovanu S, Dimitrievici LT, Dey N, Ashour AS (2018) Texture anisotropy technique in brain degenerative diseases. Neural Comput Appl 30(5):1667-1677. https://doi.org/10.1007/s00521-016-2777-7

33. Chaddad A, Tanougast C (2016) Quantitative evaluation of robust skull stripping and tumour detection applied to axial MR images. Brain Infor 3(1):53-61. https://doi.org/10.1007/s40708-016-0033-7

34. Qian X, Wang J, Guo S, Li Q (2013) An active contour model for medical image segmentation with application to brain CT image. Med Phys 40(2):021911.

35. Shriranjani D, Tebby SG, Satapathy SC, Dey N, Rajinikanth V (2018) Kapur's entropy and active contour-based segmentation and analysis of retinal optic disc. LNEE 490:287-295. https://doi.org/10.1007/978-981-10-8354-9_26

36. Rajinikanth V, Raja NSM, Satapathy SC, Fernandes SL (2017) Otsu's multi-thresholding and active contour snake model to segment dermoscopy images. J Med Imag Health In 7(8):1837-1840. https://doi.org/10.1166/jmihi.2017.2265

37. Malek et al. (2012) Seed point selection for seed-based region growing in segmenting microcalcifications, In. International Conference on Statistics in Science, Business, and Engineering (ICSSBE). https://doi.org/10.1109/ICSSBE.2012.6396580

38. Hore S, Chakraborty S, Chatterjee S, Dey N, Ashour AS, Chung LV, Le DN (2016) An integrated interactive technique for image segmentation using stack based seeded region growing and thresholding. I J Electrical Compu Engg (IJECE) 6(6):2773-2780. https://doi.org/10.11591/ijece.v6i6.11801 
39. Chan TF, Vese LA (2001)Active contours without edges. IEEE T Image Process 10(2):266277.

40. Malladi R, Sethian A, Vemuri BC (1995) Shape modeling with front propagation: A level set approach. IEEE T Pattern Anal Mac Int 17(2):158-175. https://doi.org/10.1109/34.368173

41. Vaishnavi G, Jeevananthan K, Begum SR, Kamalanand K (2014) Geometrical analysis of schistosome egg images using distance regularized level set method for automated species identification. J Bioinformatics Intell Cont 3:147-152. https://doi.org/10.1166/jbic.2014.1080

42. Shree TDV, Revanth K, Raja NSM, Rajinikanth V (2018) A hybrid image processing approach to examine abnormality in retinal optic disc. Procedia Compu Sci 125:157-164. https://doi.org/10.1016/j.procs.2017.12.022

43. Dey N, Rajinikanth V, Ashour AS, Tavares JMRS (2018) Social group optimization supported segmentation and evaluation of skin melanoma images. Symmetry 10(20):51. https://doi.org/10.3390/sym10020051

44. Satapathy S, Naik A (2016) Social group optimization (SGO): a new population evolutionary optimization technique. Complex \& Intelligent Systems 2(3):173-203.

45. Naik A, Satapathy SC, Ashour AS, Dey N (2016) Social group optimization for global optimization of multimodal functions and data clustering problems. Neural Comput Appl 30(1):271-287. https://doi.org/10.1007/s00521-016-2686-9

46. Kannappan PL (1972) On Shannon's entropy, directed divergence and inaccuracy. Probab Theory Rel Fields 22:95-100.

47. Paul S, Bandyopadhyay B *2014) A novel approach for image compression based on multilevel image thresholding using Shannon entropy and differential evolution. Students' Technology Symposium (TechSym), IEEE 56-61. https://doi.org/10.1109/TechSym.2014. 6807914

48. Roerdink JBTM, Meijster A (2001) The watershed transform: Definitions, algorithms and parallelization strategies. Fundamenta Informaticae 41:187-228.

49. Lu H, Kot AC, Shi YQ (2004) Distance-reciprocal distortion measure for binary document images. IEEE Signal Process Letter 11(2):228-231.

50. Moghaddam RF Cheriet M (2010) A multi-scale framework for adaptive binarization of degraded document images. Pat Recognition 43(6):2186-2198.

51. Beagum S, Dey N, Ashour AS, Sifaki-Pistolla D, Balas VE (2017) Nonparametric de-noising filter optimization using structure-based microscopic image classification. Microscopy research and technique 80(4):419-429. https://doi.org/10.1002/jemt.22811

52. Roy P, Goswami S, Chakraborty S, Azar AT, Dey N (2014) Image segmentation using rough set theory: a review. I J of Rough Sets and Data Analysis (IJRSDA) 1(2):62-74. https://doi.org/10.4018/ijrsda.2014070105

53. Oliveira RB, Papa JP, Pereira AS, Tavares JMRS (2018) Computational methods for pigmented skin lesion classification in images: review and future trends. Neural Comput Appl 29(3):613-636. https://doi.org/10.1007/s00521-016-2482-6

54. Tavares JMRS (2014) Analysis of biomedical images based on automated methods of image registration. LNCS 8887:21-30. https://doi.org/10.1007/978-3-319-14249-4_3

55. Haralick RM, Shanmugam K, Dinstein I (1973) Textural features of image classification. IEEE T Syst Man Cy SMC-3(6):610-621. https://doi.org/10.1109/TSMC.1973.4309314

56. Soh L, Tsatsoulis C (1999) Texture analysis of SAR sea ice imagery using gray level cooccurrence matrices. IEEE $\mathrm{T}$ GeoSci Remote $\mathrm{S}$ 37(2):780-795. https://doi.org/10.1109/36.752194

57. Clausi DA (2002) An analysis of co-occurrence texture statistics as a function of grey level quantization. Can J Remote Sensing 28(1):45-62. https://doi.org/10.5589/m02-004 
58. Kamalanand K, Ramakrishnan S (2015) Effect of gadolinium concentration on segmentation of vasculature in cardiopulmonary magnetic resonance angiograms. J Med Imag Health In 5(1):147-151. https://doi.org/10.1166/jmihi.2015.1370

59. Ma Z, Tavares JMRS (2015) A review of the quantification and classification of pigmented skin lesions: from dedicated to hand-held devices. J Medi Syst 39(11):177. https://doi.org/10.1007/s10916-015-0354-8

60. Manickavasagam K, Sutha S, Kamalanand K (2014) Development of systems for classification of different plasmodium species in thin blood smear microscopic images. J Adv Microscopy Res 9(2):86-92. https://doi.org/10.1166/jamr.2014.1194

61. Kamalanand K, Jawahar PM (2016) Comparison of particle swarm and bacterial foraging optimization algorithms for therapy planning in HIV/AIDS patients. I J Biomathematics 9(2):1650024. https://doi.org/10.1142/S1793524516500248

62. Paramasivam A, Kamalanand K (2018) An approach based on information theory for selection of systems for efficient recording of electrogastrograms. LNNS 24:463-471. https://doi.org/10.1007/978-981-10-6890-4_45

63. Sathya DJ, Geetha K (2017) Hybrid ANN optimized artificial fish swarm algorithm based classifier for classification of suspicious lesions in breast DCE-MRI. P J Med Phy Engg 23(4): 81-88. https://doi.org/10.1515/pjmpe-2017-0014

64. Yang Z, Wang Y, Ouyang G (2014) Adaptive Neuro-Fuzzy inference system for classification of background EEG signals from ESES patients and controls. The Sci World J 2014(Article ID 140863): 8. https://doi.org/10.1155/2014/140863

65. Wang et al. (2016) Image feature-based affective retrieval employing improved parameter and structure identification of adaptive neuro-fuzzy inference system. Neural Compu App 116. https://doi.org/10.1007/s00521-016-2512-4

66. Ahmed et al. (2017) Effect of fuzzy partitioning in Crohn's disease classification: A neurofuzzy-based approach. MediBio Engg Compu 55(1):101-115. https://doi.org/ 10.1007/s11517-016-1508-7

67. Yushkevich PA, Piven J, Hazlett HC, Smith RG, Ho S, Gee JC, Gerig G (2006) User-guided 3D active contour segmentation of anatomical structures: Significantly improved efficiency and reliability. Neuroimage 31(3):1116-28.

68. ITK-SNAP (http://www.itksnap.org/pmwiki/pmwiki.php)

69. https://proscans.in 
Manuscript Title: Social-Group-Optimization based Tumor Evaluation Tool for Clinical Brain MRI of Flair/DW Modality

Conflict of interest: The authors declare that they have no conflict of interest 